\title{
Domain wall motion in ferromagnetic layers
}

\author{
Christof Melcher \\ School of Mathematics, University of Minnesota \\ melcher@math.umn.edu
}

\begin{abstract}
We consider the dynamics of one-dimensional micromagnetic domain walls in layers of uniaxial anisotropy. In the regime of bulk materials, i.e. when the thickness is assumed to be infinite, and the magnetostatic interaction terms appear as local quantities, explicit traveling wave solutions for the corresponding Landau-Lifshitz equation, known as Walker exact solutions, can be constructed. A natural question is whether this construction can be perturbed to the non-local regime of layers of finite thickness. Our stability analysis gives an affirmative answer.
\end{abstract}

\section{Introduction}

A static micromagnetic domain wall is an energetically optimal transition layer that separates domains of almost constant magnetization $\mathbf{m} \in \mathbb{S}^{2}$ within a ferromagnetic sample. Among the most simple examples we have parameterized transitions from an equilibrium state $(0,-1,0)$ into its antipodal counterpart $(0,1,0)$ to be attained at $\pm \infty$, respectively. The phrase equilibrium state indicates that the end-states are supposed to point towards the so-called easy axis, i.e. having a direction which is preferred by crystalline anisotropy.

When an external magnetic field $h=H \hat{\mathbf{e}}_{2}$ is applied, that is supposed to point towards the easy axis, the end-states are no longer equally preferred: The domain wall is expected to become unstable and starts to move. According to [9], the evolution of magnetization distributions is characterized by the Landau-Lifshitz 
equations that describe a damped precession of the magnetization vector $\mathbf{m}$ :

$$
\begin{aligned}
& \mathbf{m} \wedge \partial_{t} \mathbf{m}+\alpha \partial_{t} \mathbf{m}+\nabla E_{\kappa}(\mathbf{m})(1-\mathbf{m} \otimes \mathbf{m})=h(1-\mathbf{m} \otimes \mathbf{m}) \\
& \mathbf{m}: \mathbb{R} \times \mathbb{R} \rightarrow \mathbb{S}^{2} \quad \text { with } \quad \mathbf{m}(x, \pm \infty)=(0, \pm 1,0) \text { for } x \in \mathbb{R} .
\end{aligned}
$$

The functional $E_{\kappa}(\mathbf{m})$ is the (reduced) internal micromagnetic energy (to be introduced later). The number $\kappa \geq 0$ is a dimensionless parameter that measures the inverse of the relative thickness of the sample. The micromagnetic energy depends on $\mathbf{m}$ in a non-local fashion, since it involves in particular the magnetostatic energy that relates to $\mathbf{m}$ via Maxwell's equations. Nevertheless it turns out that for one-dimensional domain walls in case of bulk materials, i.e. if $\kappa=0$, the correspondence is entirely local.

The static domain wall problem in the absence of applied fields, i.e. the optimal profile problem of a micromagnetic domain wall, was extensively studied and is relatively well understood, see [6], [4], [10]: In the bulk regime (for $\kappa=0$ ) the corresponding variational principle is always local and (essentially) equivalent to the optimal profile problem for the standard Ginzburg-Landau functional. In particular it exhibits rapidly decaying transition profiles. It is also known that (for $\kappa>0$ ) magnetostatic interaction enforces a very slow decay (logarithmic tails) of transition profiles, especially in the regime of thin films, i.e. if $\kappa \geq 1$, see [10].

Regarding the dynamic problem, a special class of solutions to constant coefficient systems like (6) are traveling wave solutions, i.e. solutions of the form $\mathbf{m}=\mathbf{m}(x+c t)$, that describe a motion of constant speed $c$. In case $\kappa=0$ the traveling wave ansatz turns (1) into a constrained system of non-linear ODEs. Surprisingly, these equations can be solved explicitly. The solutions are referred to as Walker exact solutions, see [11], [6]. Our goal is to show that this situation is indeed generic and can be perturbed to layers of large but finite diameter. As this corresponds to the case of small non-local interaction, the non-local character of the equations will play a minor role. We will show that, in suitable coordinates, domain wall motion according to Landau-Lifshitz dynamics fits into the context of non-local, weakly coupled reaction-diffusion systems.

Our approach is motivated by previous discussions of traveling waves for nonlocal models of phase transitions, see e.g. [1]. Existence, uniqueness, and stability questions were investigated for non-local scalar reaction-diffusion equations emerging as $L^{2}$-gradient flows. Still, the present situation differs in some fundamental regards: First we have to deal with a system. Moreover the non-locality 
appears as a non-linear term. Nevertheless our perturbation argument is inspired by the strategies developed in [1].

\section{The micromagnetic energy and dynamics}

Let us briefly recall the dimensionally reduced micromagnetic energy associated with one-dimensional domain wall problems. We refer to [6], [2] for a general discussion of micromagnetic models and to [6], [4], [10] for a more detailed description of one-dimensional domain wall models. For an infinitely extended layer and magnetization fields $\mathbf{m}: \mathbb{R} \rightarrow \mathbb{S}^{2}$ we have, after rescaling by the exchange length, the dimensionally reduced energy functional

$$
E_{\kappa}(\mathbf{m})=\frac{1}{2} \int\left|\mathbf{m}^{\prime}\right|^{2} d x+\frac{1}{2} \int\left(1-m_{2}^{2}\right) d x+\int \mathbf{e}_{\operatorname{mag}}^{\kappa}(\mathbf{m}) d x .
$$

The Dirichlet term, the so-called exchange energy, comes from quantum mechanical spin interaction. The second term models crystalline anisotropy and characterizes the so-called easy axis $\mathbb{R} \hat{\mathbf{e}}_{2}$. We have set the balancing factor (quality factor) 1 . Finally the (reduced) magnetostatic interaction given in terms of Fourier multiplication has the following form:

$$
\mathbf{e}_{\operatorname{mag}}^{\kappa}(\mathbf{m})=\frac{1}{2}\left(\sigma(D / \kappa) m_{1} \cdot m_{1}\right)+\frac{1}{2}\left(m_{3}^{2}-\sigma(D / \kappa) m_{3} \cdot m_{3}\right) .
$$

The operator $u \mapsto \sigma(D / \kappa) u$ is defined by $\mathcal{F}^{*}(\sigma(\xi / \kappa) \hat{u})$, where $\mathcal{F}^{*}$ denotes the inverse Fourier transform. This operator can equivalently be described by a convolution kernel; see [4], [10] for a detailed treatment. This density arises from dimensional reduction of the 3D magnetostatic energy density $\frac{1}{2}|\mathcal{H}(\mathbf{m})|^{2}$, where the induced magnetostatic fields $\mathcal{H}(\mathbf{m})$ is formally given by the Helmholtz projection $\nabla \Delta^{-1} \nabla \cdot \mathbf{m}$ of $\mathbf{m}$. The Fourier multiplier $\sigma(\xi)$ is a Lipschitz continuous function given by

$$
\sigma(\xi)=1-\frac{1-\exp (-2|\xi|)}{2|\xi|} \sim\left\{\begin{array}{cl}
|\xi| & \text { for low frequencies } \xi \\
1 & \text { for high frequencies } \xi
\end{array}\right.
$$

The dimensionless parameter $\kappa$ is the quotient of the exchange length over the relative film thickness, and we suppose $\kappa$ to be small. The associated variational principle for a static domain wall

$$
E_{\kappa}(\mathbf{m}) \rightarrow \min \quad \text { for } \quad \mathbf{m}: \mathbb{R} \rightarrow \mathbb{S}^{2} \text { with } \mathbf{m}( \pm \infty)=(0, \pm 1,0)
$$


is non-convex through the constraint $|\mathbf{m}|=1$ and the anisotropy contribution, and non-local through the magnetostatic interaction. Still the existence of minimizers is ensured by the direct method of the calculus of variations.

The dynamic transition from $\mathbf{m}(-\infty)=(0,-1,0)$ to $\mathbf{m}(\infty)=(0,1,0)$ is assumed to be driven by a constant applied field $h$ pointing towards one of the endstates, i.e. for some constant field strength $H$, it is supposed to be $h=H \hat{\mathbf{e}}_{2}$. Given the internal micromagnetic energy $E_{\kappa}(\mathbf{m})$ on saturated vector-fields the Landau-Lifshitz dynamic (see [9], [6]) of $\mathbf{m}$ is described by the following system of equations

$$
\mathbf{m} \wedge \partial_{t} \mathbf{m}+\alpha \partial_{t} \mathbf{m}+\nabla E_{\kappa}(\mathbf{m})(1-\mathbf{m} \otimes \mathbf{m})=h(1-\mathbf{m} \otimes \mathbf{m}),
$$

where $\mathbf{a} \wedge \mathbf{b}$ is denotes the vector-product for $\mathbf{a}, \mathbf{b} \in \mathbb{R}^{3}$. This is a cross-over between a Hamiltonian and dissipative system. The coefficient $\alpha>0$ is referred to as the Gilbert damping factor.

The local situation without magnetostatic interaction. The energy density arising from anisotropy and the applied field in combination gives rise to a doublewell potential

$$
W(\mathbf{m})=\frac{1}{2}\left(1-m_{2}^{2}\right)-H m_{2},
$$

when combined with the constraint $|\mathbf{m}|=1$. In particular, $H=0$ corresponds to equal depth, and there is a static connection of the end-states characterized by the variational principle (5). For $0<H<1$ we still have bi-stable character. In fact when we introduce spherical polar coordinates $(\varphi, \theta) \in[0,2 \pi) \times\left(-\frac{\pi}{2}, \frac{\pi}{2}\right)$ such that the end-states are attained at $\theta= \pm \frac{\pi}{2}$, then for fixed fixed polar angle $\varphi$ the function

$$
\theta \mapsto W(\mathbf{m}[\varphi, \theta]) \quad \text { is bi-stable for }|H|<1,
$$

meaning that for the extended function the (local) minima to be attained at $\theta= \pm \frac{\pi}{2}$ are non-degenerate. The exchange term $\frac{1}{2}\left|\mathbf{m}^{\prime}\right|^{2}$ can be considered as an analog for the kinetic energy density in thermo-dynamical models. Note that e.g. for constant polar angel $\varphi$ we have $\frac{1}{2}\left|\mathbf{m}^{\prime}\right|^{2}=\frac{1}{2}\left|\theta^{\prime}\right|^{2}$. Thus, the $L^{2}$-gradient flow of $\frac{1}{2}\left|\mathbf{m}^{\prime}\right|^{2}+W(\mathbf{m})$ under the additional constraint of constant polar inclinations yields a standard type of reaction-diffusion equations in terms of the azimuthal angle

$$
\theta_{t}=\theta_{x x}-f(\theta) \quad \text { with } \quad f(\theta)=\frac{\partial}{\partial \theta} W(\mathbf{m}[\varphi, \theta]) .
$$


Equations of that type are widely studied and well understood, see e.g. [3]. One can in particular show the existence of a unique propagation speed $c$ and a monotone traveling wave profile $\theta=\theta(x+c t)$ solving the parabolic equation.

Limiting form of non-local interaction. We have the following limiting behavior for the bulk regime $(\kappa=0)$ and the thin-film regime $(\kappa \rightarrow \infty)$, respectively

$$
\sigma(D / \kappa) u \rightarrow u \text { as } \kappa \downarrow 0 \text { and } \kappa \sigma(D / \kappa) u \rightarrow(-\Delta)^{1 / 2} u \text { as } \kappa \uparrow \infty .
$$

We observe a transition from a zero-order elliptic operator to a first-order elliptic operator. In suitable function spaces the correspondence $\kappa \mapsto \sigma(D / \kappa) u$ is continuous for $\kappa \in[0,1]$ but not uniformly differentiable. This comes from the fact $\sigma(0)=0$ so that $\sigma(D / \kappa) 1=0$, but in the bulk limit $\kappa \downarrow 0$ we have $\sigma(\xi / \kappa) \rightarrow 1$ for all $\xi \neq 0$. We observe a singular change of action on very low frequencies. In this regard the non-local action can be considered as a singular perturbation with small parameter $\kappa$. This change of analytic structure potentially gives rise to a change in the decay behavior of transition profiles, which was verified in the equilibrium case, see [10]. Observe that for $\kappa=0$ we have by means of (3) an entirely local density for the internal energy. In fact

$$
E_{0}(\mathbf{m})=\frac{1}{2} \int\left|\mathbf{m}^{\prime}\right|^{2} d x+\frac{1}{2} \int\left(1-m_{2}^{2}+m_{1}^{2}\right) d x .
$$

It is well know that in this limiting case and in the absence of applied fields the static domain wall problem, referred to as Bloch wall, can be solved explicitly, a calculation that goes back Landau and Lifshitz [9]. It it also well known that in this limiting regime explicit traveling wave solutions for (6), i.e. solutions of

$$
c \mathbf{m} \wedge \partial_{x} \mathbf{m}+c \alpha \partial_{x} \mathbf{m}+\nabla E_{0}(\mathbf{m})(1-\mathbf{m} \otimes \mathbf{m})=h(1-\mathbf{m} \otimes \mathbf{m}),
$$

for a certain propagation speed depending on $H$ can be found, a construction that goes back to Walker, see [11],[6].

The perturbation result. Let us consider (6) for $\kappa>0$. Our goal is to show that the situation described above is, considered in a suitable coordinate system, not degenerate and can be perturbed at least for small $H$ and small $\kappa$, i.e. for small applied fields and when the sample is sufficiently thick. More precisely we prove the following:

Theorem. There is a number $H_{0}>0$ such that for every $|H|<H_{0}$ there is a number $\kappa(H)>0$ such that for all $\kappa<\kappa(H)$ there is a traveling wave solution for the Landau-Lifshitz equations connecting antipodal states along the easy axis. 
We show that in properly chosen coordinates the linearized problem can be considered as a weakly coupled $2 \times 2$ system that we will show to be non-degenerate. But we first review briefly how to solve the static problem and the traveling wave problem in the local bulk regime.

\section{Bloch wall like equilibria}

The micromagnetic Bloch wall, which is observed in the bulk regime, is the most basic one-dimensional domain wall construction: it is characterized by a rotation perpendicular to the transition axis. A rotation field like that does not induce magnetic charge, i.e. $\operatorname{div} \mathbf{m}=0$, thus no magnetostatic interaction. In view of dynamic considerations it turns out to be necessary to consider the slightly more general situation of constant polar inclination, which induces magnetostatic interaction but in local correspondence with the magnetization field.

We fix a geodesic arc connecting the equilibrium states $m_{2}= \pm 1$ by choosing a fixed polar angle $\varphi$. Note that $\varphi=0$ corresponds to the Bloch wall. In the absence of external fields in the bulk limit, the energetically optimal transition profile associated with this connection is (up to translations) characterized by the constrained variational principle

$$
\begin{aligned}
E_{0}(\mathbf{m}) & =\frac{1}{2} \int\left|\mathbf{m}^{\prime}\right|^{2} d x+\frac{1}{2} \int\left(1-m_{2}^{2}+m_{1}^{2}\right) d x \rightarrow \min \\
\mathbf{m}: \mathbb{R} & \rightarrow \mathbb{S}^{2} \text { with } m_{1}=m_{3} \tan \varphi \text { and } m_{2}( \pm \infty)= \pm 1
\end{aligned}
$$

Note that the boundary condition at infinity can be substituted by e.g. $m_{2}(0)=0$ that clearly enforces a non-trivial transition for finite energy. Then a standard equipartition argument yields, for a minimizer, the following optimality relation:

$$
\left|\mathbf{m}^{\prime}\right|^{2}=1-m_{2}^{2}+m_{1}^{2}=\left(1+\sin ^{2} \varphi\right)\left(1-m_{2}^{2}\right) ;
$$

This relation is admissible regarding the constraints: indeed for a monotone component $m_{2}$ (to be justified a posteriori) we have $\left|\mathbf{m}^{\prime}\right|=m_{2}^{\prime} /\left(1-m_{2}\right)^{1 / 2}$, thus

$$
m_{2}^{\prime}=\left(1+\sin ^{2} \varphi\right)^{1 / 2}\left(1-m_{2}^{2}\right) \quad \text { with } \quad m_{2}(0)=0
$$

which is solved by the monotonically increasing function

$$
m_{2}(x)=\tanh \left[\left(1+\sin ^{2} \varphi\right)^{1 / 2} x\right] .
$$


In view of the constraints, the profiles $m_{1}(x)$ and $m_{3}(x)$ are given by

$$
\sin \varphi \operatorname{sech}\left[\left(1+\sin ^{2} \varphi\right)^{1 / 2} x\right] \text { and } \cos \varphi \operatorname{sech}\left[\left(1+\sin ^{2} \varphi\right)^{1 / 2} x\right] .
$$

For $\varphi \in[0, \pi / 2)$ the corresponding magnetization fields agree up to a scaling factor and a rotation in the $\hat{\mathbf{e}}_{1} \times \hat{\mathbf{e}}_{3}$ plane.

\section{Traveling wave ansatz and Walker's construction}

Let us turn to the dynamic situation governed by the Landau-Lifshitz equation

$$
\mathbf{m} \wedge \partial_{t} \mathbf{m}+\alpha \partial_{t} \mathbf{m}+\nabla E_{\kappa}(\mathbf{m})(1-\mathbf{m} \otimes \mathbf{m})=h \cdot(1-\mathbf{m} \otimes \mathbf{m}) .
$$

With the traveling wave ansatz $\mathbf{m}=\mathbf{m}(x+c t)$ for a constant propagation speed $c$ we get

$$
c \mathbf{m} \wedge \mathbf{m}^{\prime}+\alpha c \mathbf{m}^{\prime}+\nabla E_{\kappa}(\mathbf{m})(1-\mathbf{m} \otimes \mathbf{m})=h \cdot(1-\mathbf{m} \otimes \mathbf{m})
$$

In case of finite internal energy $E_{\kappa}(\mathbf{m})<\infty$ and vanishing energy density at infinity, we can multiply the equation by $\mathbf{m}^{\prime}$ to get the following formula for the propagation speed

$$
\frac{c}{2} \int\left|\mathbf{m}^{\prime}\right|^{2} d x=\frac{H}{\alpha} .
$$

Thus the propagation speed is related with the strength of the applied field and the exchange energy of a traveling wave profile.

\section{Walker solutions}

We recall the construction of explicit solution for (15), the so-called Walker exact solutions, see [11], [6]. The original calculations become more transparent, when the equation is considered in the canonical orthogonal frame $\left\{\mathbf{m}^{\prime}, \mathbf{m} \wedge \mathbf{m}^{\prime}\right\}$ on the tangent space of $\mathbb{S}^{2}$ along $\mathbf{m}$. Here we assume that $\left|\mathbf{m}^{\prime}\right|>0$ to be justified a posteriori.

Walker's first assumption: The transition proceeds on a fixed hyper-plane, i.e. $\mathbf{m} \wedge \mathbf{m}^{\prime}=\left|\mathbf{m}^{\prime}\right| \nu$ for some fixed unit vector $\nu$ perpendicular to the applied field $h$. This setting is analogous to the static problem with constant inclination. Then $\mathbf{m}$ 
is given by a fixed polar angle $\varphi$ and a two-dimensional vector field taking values in $\mathbb{S}^{1}$ embedded in this hyper-plane, i.e. in coordinates

$$
\mathbf{m}=\left[\begin{array}{c}
\left(1-m_{2}^{2}\right)^{1 / 2} \sin \varphi \\
m_{2} \\
\left(1-m_{2}^{2}\right)^{1 / 2} \cos \varphi
\end{array}\right] .
$$

Walker's second assumption: The driving force is totally compensated by the dissipative term, i.e., since in view of the first assumption $h=H \hat{\mathbf{e}}_{2} \perp \mathbf{m} \wedge \mathbf{m}^{\prime}$, after multiplying (15) by $\mathbf{m}^{\prime}$ we have

$$
\alpha c\left|\mathbf{m}^{\prime}\right|^{2}=h \cdot \mathbf{m}^{\prime} \text { and } \nabla E(\mathbf{m}) \cdot \mathbf{m}^{\prime}=0 .
$$

A simple calculation using (17) shows that

$$
h \cdot \mathbf{m}^{\prime}=H\left(1-m_{2}^{2}\right)^{1 / 2}\left|\mathbf{m}^{\prime}\right|
$$

thus

$$
\alpha c\left|\mathbf{m}^{\prime}\right|=H\left(1-m_{2}^{2}\right)^{1 / 2} .
$$

The second relation $\nabla E(\mathbf{m}) \cdot \mathbf{m}^{\prime}=0$ is the Euler-Lagrange equation for a static Bloch wall type transition, as considered in the last section, which implies the optimality relation

$$
\left|\mathbf{m}^{\prime}\right|=\left(1+\sin ^{2} \varphi\right)^{1 / 2}\left(1-m_{2}^{2}\right)^{1 / 2} .
$$

Walker's third assumption: The precession determines the inclination: We multiply the equation (15) by $\mathbf{m} \wedge \mathbf{m}^{\prime}$ and get

$$
\nabla E(\mathbf{m}) \cdot \mathbf{m} \wedge \mathbf{m}^{\prime}=c\left|\mathbf{m}^{\prime}\right|^{2} .
$$

Since in view of (17) $\mathbf{m}^{\prime} \wedge \mathbf{m}=\left|\mathbf{m}^{\prime}\right|\left(1-m_{2}^{2}\right)^{-1 / 2} \partial_{\varphi} \mathbf{m}$ we have

$$
\begin{aligned}
\nabla E(\mathbf{m}) \cdot \mathbf{m} \wedge \mathbf{m}^{\prime} & =-\left|\mathbf{m}^{\prime}\right|\left(1-m_{2}^{2}\right)^{-1 / 2} \frac{\partial}{\partial \varphi} E(\mathbf{m}) \\
& =-\frac{1}{2}\left|\mathbf{m}^{\prime}\right| \sin (2 \varphi)\left(1-m_{2}^{2}\right)^{1 / 2}
\end{aligned}
$$

Hence (20) can be written as

$$
c\left|\mathbf{m}^{\prime}\right|=\frac{1}{2} \sin (2 \varphi)\left(1-m_{2}^{2}\right)^{1 / 2}
$$

We observe that equations (18), (19), and (21) are compatible and can be solved jointly. The resulting restrictions on parameters show, in view of the results of the last section, that the Walker solution has the following properties: 
(a) The profile $m_{2}: \mathbb{R} \rightarrow[-1,1]$ agrees with (13) and is monotonically increasing.

(b) The inclination angle of the Walker path is given by $\frac{1}{2} \sin (2 \varphi)=H / \alpha$.

(c) The propagation speed is given by $c_{0}=\frac{1}{2} \sin (2 \varphi) /\left(1+\sin ^{2} \varphi\right)^{1 / 2}$.

Note that there is a maximal field strength $H^{*}>0$ beyond which this construction breaks down. But for all admissible $|H|<H^{*}$ the corresponding Walker solutions agree up to a scaling factor and a constant rotation in the $\hat{\mathbf{e}}_{1} \times \hat{\mathbf{e}}_{3}$ plane.

\section{Formulation in adapted coordinates}

The goal is to formulate the traveling wave problem in a suitable coordinate system that allows one to apply perturbation methods. More specifically, we aim for a formulation in terms of planar vector fields

$$
z=(u, v): \mathbb{R} \rightarrow \mathbb{R}^{2} \text { with } z( \pm \infty)=(0, \pm 1),
$$

where $(0, \pm 1)$ should correspond to the end-states $(0, \pm 1,0)$. Moreover, in these coordinates and for fixed applied field $H$, the path of the associated Walker solution should be given by the line segment

$$
\{(u, v): u=0, v \in[-1,1]\} .
$$

For this purpose we first choose stereographic coordinates on $\mathbb{S}^{2}$ followed by a (polar) rotation of the standard frame in $\mathbb{R}^{3}$ by the inclination angle that corresponds to the applied field.

Let $\mathbf{m}$ represent the magnetization field in the canonical frame induced by the orientation of the magnetic layer in $\mathbb{R}^{3}$. We introduce a second director field $\mathbf{m}^{*}$ that differs from $\mathbf{m}$ only by a fixed rotation $\mathrm{R} \in \mathrm{SO}(3)$, i.e. $\mathbf{m}=\mathbf{R} \mathbf{m}^{*}$.

Recall that for any direction $\mathbf{m}^{*} \in \mathbb{S}^{2} \backslash\left\{-\hat{\mathbf{e}}_{3}\right\}$ we have stereographic coordinates $z=(u, v)$ and the following parameterization

$$
z=(u, v) \mapsto \mathbf{m}^{*}[z]=\lambda(z)\left[\begin{array}{c}
u \\
v \\
\frac{1-|z|^{2}}{2}
\end{array}\right] \quad \text { with } \quad \lambda(z)=\frac{2}{1+|z|^{2}}
$$


The function $\lambda(z)$ is referred to as the conformal factor, and the induced metric tensor reads as $\lambda^{2}(z) \delta_{\alpha \beta}$.

For fixed polar angle $\varphi$ we introduce the rotation matrix $\mathrm{R}=\mathrm{R}(\varphi)$

$$
\mathrm{R}=\left[\begin{array}{ccc}
\cos \varphi & 0 & -\sin \varphi \\
0 & 1 & 0 \\
\sin \varphi & 0 & \cos \varphi
\end{array}\right] \in \operatorname{SO}(3)
$$

Note that $\mathrm{R}$ stabilizes the end-states pointing towards the easy axis $\mathbb{R} \hat{\mathbf{e}}_{2}$. Finally we get the following composite parameterization for $\mathbf{m}$

$$
z \mapsto \mathbf{m}^{*}[z] \mapsto \mathbf{R} \mathbf{m}^{*}[z]=\mathbf{m}[z] \text { that maps } \mathbb{R}^{2} \rightarrow \mathbb{S}^{2} \backslash\left\{-\mathrm{R} \hat{\mathbf{e}}_{3}\right\}
$$

Note that the parameterizations $z \mapsto \mathbf{m}^{*}[z]$ and $z \mapsto \mathbf{m}[z]$ have the same metric:

$$
\frac{\partial \mathbf{m}}{\partial z_{\alpha}} \cdot \frac{\partial \mathbf{m}}{\partial z_{\beta}}=\frac{\partial \mathbf{m}^{*}}{\partial z_{\alpha}} \cdot \frac{\partial \mathbf{m}^{*}}{\partial z_{\beta}}=\lambda^{2}(z) \delta_{\alpha \beta} .
$$

In this setting we have the freedom of choosing $\mathrm{R}$. Thus, we gauge this chart according to Walker's construction: If $(\mathbf{m}, c)$ is a Walker solution corresponding to the field strength $H$, then there is a polar angle $\varphi=\varphi(H)$ and a corresponding rotation $\mathrm{R}=\mathrm{R}(\varphi)$ such that $\mathbf{m}^{*}=\mathbf{R}^{*} \mathbf{m}$ has the canonical form $\mathbf{m}^{*}=\left(0, m_{2}^{*}, m_{3}^{*}\right)$ of a standard Bloch wall. Then the stereographic projection of $\left(0, m_{2}^{*}, m_{3}^{*}\right)$ agrees with the line segment (22). Thus the Walker solution has a representation $\mathbf{m}_{0}=$ $\mathbf{m}\left[z_{0}\right]$ where $z_{0}=\left(0, v_{0}\right)$ for some monotonically increasing function $v_{0}$. Note that all such $\mathbf{m}^{*}$ arising from a Walker solution agree up to a scaling factor. More specifically, we found in Section 4 that $m_{2}=m_{2}^{*}$ is given by the Bloch wall like equilibrium (13), i.e. $m_{2}(x)=\tanh \left[\left(1+\sin ^{2} \varphi\right)^{1 / 2} x\right]$. But in our chart we have $m_{2}=\lambda\left(z_{0}\right) v_{0}$, and a simple calculation shows that

$$
v_{0}(x)=\tanh \left[\frac{1}{2}\left(1+\sin ^{2} \varphi\right)^{1 / 2} x\right] .
$$

In particular $v_{0}$ and its derivatives share similar asymptotic properties with the original Walker profile.

\section{Transformation of Landau-Lifshitz equations}

In stereographic coordinates, the dissipation $\alpha \partial_{t} \mathbf{m}$ and the precession $\mathbf{m} \wedge \partial_{t} \mathbf{m}$ can be written in complex notation as $\alpha \lambda^{2}(z) \partial_{t} z$ and $i \lambda^{2}(z) \partial_{t} z$, or in vector 
notation as $\alpha \lambda^{2}(z) \partial_{t} z$ and $\lambda^{2}(z) \partial_{t} z^{\perp}$, where " $\perp$ " denotes the $\frac{\pi}{2}$-rotation of a 2D-vector. In particular, along the line segment $\{v \in[-1,1], u=0\} \subset \mathbb{R}^{2}$ describing the Walker path, we have the convenient form of time derivatives

$$
\alpha \partial_{t} z+\partial_{t} z^{\perp}=\alpha\left(\begin{array}{c}
0 \\
v_{t}
\end{array}\right)-\left(\begin{array}{c}
v_{t} \\
0
\end{array}\right)
$$

According to (6), the transformed 1D-Landau-Lifshitz equations have the form

$$
\lambda^{2}(z)\left(\alpha \partial_{t} z+i \partial_{t} z\right)+\frac{\partial \mathbf{m}}{\partial z} \cdot \nabla E_{\kappa}(\mathbf{m})=\frac{\partial \mathbf{m}}{\partial z} \cdot h
$$

where $\mathbf{m}=\mathbf{m}[z]$ is parameterized by $z$. After multiplying this equation by $\lambda^{-2}(z)$, the traveling wave ansatz $z=z(x+c t)$ suggests introducing a nonlinear function of the form

$$
G((z, c), \kappa)=c \partial_{x}\left(\alpha z+z^{\perp}\right)-D_{x} \partial_{x} z+F(z)+B(\kappa, z),
$$

where $\lambda^{-2}(z) \nabla_{z} \mathbf{m} \cdot\left(\nabla E_{\kappa}(\mathbf{m})-h\right)=-D_{x} \partial_{x} z+F(z)+B(\kappa, z)$. This function should characterize the traveling wave solutions as the solution of $G((z, c), \kappa)=$ 0 . Let us discuss the above terms in some detail. The second order terms arising from exchange energy can be written as

$$
\lambda^{-2}(z) \frac{\partial \mathbf{m}}{\partial z} \cdot \partial_{x}^{2} \mathbf{m}[z]=D_{x} \partial_{x} z,
$$

where, for a vector field $z: \mathbb{R} \rightarrow \mathbb{R}^{2}$ which parameterizes the magnetization field $\mathbf{m}: \mathbb{R} \rightarrow \mathbb{S}^{2}$,

$$
D_{x} \partial_{x} z=\partial_{x}^{2} z+\Gamma(z)\left\langle\partial_{x} z, \partial_{x} z\right\rangle
$$

denotes the covariant $x$-derivative (with respect to the metric $\lambda^{2}(z)$ ) of $\partial_{x} z$ along $z$. We have the geometric terms

$$
\Gamma(z)\left\langle\partial_{x} z, \partial_{x} z\right\rangle=\lambda(z)\left|\partial_{x} z\right|^{2} z-2 \lambda(z)\left(z \cdot \partial_{x} z\right) \partial_{x} z .
$$

We combine the terms coming from anisotropy and the applied field in the function

$$
F(z)=-\frac{1}{2} \lambda^{-2}(z) \nabla_{z}\left(m_{2}^{2}[z]+2 H m_{2}[z]\right) .
$$

Finally the magnetostatic interaction term is given by

$$
B(\kappa, z)=\lambda^{-2}(z)\left(\frac{\partial m_{1}}{\partial z} \sigma(D / \kappa) m_{1}[z]+\frac{\partial m_{3}}{\partial z}(1-\sigma(D / \kappa)) m_{3}[z]\right) .
$$


Recall that the multiplier $\sigma(\xi / \kappa)$ converges to 1 pointwise as $\kappa \downarrow 0$. Thus we formally have

$$
F(z)+B(0, z)=\frac{1}{2} \lambda^{-2}(z) \nabla_{z}\left(m_{1}^{2}[z]-m_{2}^{2}[z]-2 H m_{2}[z]\right) .
$$

We shall show in the next section that the operator $z \mapsto B(\kappa, z)$ can be continuously extended to $\kappa=0$.

Let us derive a more concrete form of (31) in terms of the coordinate functions $u$ and $v$. Recall that $\mathbf{m}[z]=\mathbf{R}(\varphi) \mathbf{m}^{*}[z]$, and in particular

$$
m_{1}[z]=\cos \varphi m_{1}^{*}[z]+\sin \varphi m_{3}^{*}[z]
$$

But since by property (b) of Walker solutions (see Section 4) we have $\sin \varphi=$ $\mathcal{O}(H)$, we conclude that $m_{1}[z]=\lambda(z) u+\mathcal{O}(H)$. Hence

$$
\begin{aligned}
F(z)+B(0, z) & =\frac{1}{2} \lambda^{-2}(z) \nabla_{z}\left(\lambda^{2}(z)\left(u^{2}-v^{2}\right)\right)+\mathcal{O}(H) \\
& =\frac{1}{2} \lambda(z)\left[\begin{array}{c}
u\left(1-u^{2}+3 v^{2}\right) \\
-v\left(1-v^{2}+3 u^{2}\right)
\end{array}\right]+\mathcal{O}(H)
\end{aligned}
$$

Note that $G$ is translation invariant in the sense that if $G((z, c), \kappa)=0$ then $G\left(\left(z\left(\cdot+x_{0}\right), c\right), \kappa\right)=0$ for any $x_{0} \in \mathbb{R}$. To eliminate this source of degeneracy, we introduce an extended function $\mathcal{G}((z, c), \kappa)$. Moreover, this function should act on perturbations of the Walker solution for $\kappa=0$. Then the traveling wave problem can be formulated as follows: For given $\kappa>0$, find a perturbation $z=(u, v): \mathbb{R} \rightarrow \mathbb{R}^{2}$ for the Walker solution such that $z( \pm \infty)=(0,0)$ and a corresponding perturbation of the propagation speed $c \in \mathbb{R}$ such that

$$
\mathcal{G}((z, c), \kappa)=\left[G\left(\left(z_{0}+z, c_{0}+c\right), \kappa\right), v(0)\right]=0 .
$$

Note that $\mathcal{G}$ can be considered as a family of functions parameterized over the set of field strengths $H$ that are admissible for the Walker construction. It turns out that at least for small $H$, we can find solutions $\mathcal{G}\left(\left(z_{\kappa}, c_{\kappa}\right), \kappa\right)=0$ when $0<\kappa<$ $\kappa(H)$.

\section{Continuity and differentiability of $\mathcal{G}$}

As perturbation spaces we introduce the following Hilbert spaces

$$
X=H^{2}\left(\mathbb{R} ; \mathbb{R}^{2}\right) \times \mathbb{R} \text { and } Y=L^{2}\left(\mathbb{R} ; \mathbb{R}^{2}\right) \times \mathbb{R} .
$$


Then the mapping can be considered as an operator $\mathcal{G}: X \times \mathbb{R} \rightarrow Y$. Note that since the Fourier multiplier $\sigma(\xi)$ is symmetric, we can formally extend the parameter space to the real line. We shall show that this operator is well defined and continuous. Moreover we show that the derivative with respect to the first component $(z, c)$

$$
\mathbf{D}_{1} \mathcal{G}: X \times \mathbb{R} \rightarrow \mathcal{L}(X ; Y), \quad((z, c), \kappa) \mapsto \mathbf{D}_{1} \mathcal{G}((z, c), \kappa)
$$

is continuous. This regularity hypothesis is sufficient for a continuous version of the implicit function theorem.

Since $H^{2}(\mathbb{R}) \hookrightarrow C_{0}^{0}(\mathbb{R})$, the linear mapping $z=(u, v) \mapsto v(0)$ makes sense and is bounded, thus it is smooth. Therefore we only need to consider the function given by

$$
((z, c), \kappa) \mapsto G\left(\left(z_{0}+z, c_{0}+c\right), \kappa\right) .
$$

This function depends linearly on $c$, and this dependence is obviously smooth. We denote for a non-linear operator $N=N(z): H^{2}(\mathbb{R}) \rightarrow L^{2}(\mathbb{R})$ the Frechet derivative with respect to $z$ by $\mathbf{D} N(z) \in \mathcal{L}\left(H^{2}(\mathbb{R}) ; L^{2}(\mathbb{R})\right)$ as a bounded linear operator $\zeta \mapsto \mathbf{D} N(z)\langle\zeta\rangle$.

The Walker solution $z_{0}=\left(0, v_{0}\right)$ does not belong to the perturbation space $H^{2}\left(\mathbb{R} ; \mathbb{R}^{2}\right)$ since the function $v_{0}$ does not decay. Still $z_{0}$ is bounded and $\partial_{x} z_{0} \in H^{1}\left(\mathbb{R} ; \mathbb{R}^{2}\right)$. We infer that the covariant second derivative $D_{x} \partial_{x} z_{0}$ given by (28) is an $L^{2}$ function. Then standard estimates show that the perturbation

$$
H^{2}\left(\mathbb{R} ; \mathbb{R}^{2}\right) \ni z \mapsto D_{x}\left(\partial_{x} z_{0}+\partial_{x} z\right) \in L^{2}\left(\mathbb{R} ; \mathbb{R}^{2}\right)
$$

induces a smooth mapping, since $(z, p) \mapsto \Gamma(z)\langle p, p\rangle$ is smooth. In particular the functional derivative

$$
z \mapsto \mathbf{D}\left(D_{x}\left(\partial_{x} z_{0}+\partial_{x}(\cdot)\right)\right)(z)
$$

is continuous from $H^{2}(\mathbb{R})$ to $\mathcal{L}\left(H^{2}(\mathbb{R}) ; L^{2}(\mathbb{R})\right)$.

Next we consider the terms arising from anisotropy and the applied field. A short calculation shows that (29) can be written as

$$
F(z)=\frac{1}{2}(\lambda(z) v+H)\left[\begin{array}{c}
2 u v \\
v^{2}-u^{2}-1
\end{array}\right] .
$$


Since for the Walker solution $z_{0}=\left(0, v_{0}\right)$ we have that $v_{0}^{2}(x) \rightarrow 1$ rapidly as $|x| \rightarrow \infty$, we obviously have that $F\left(z_{0}\right) \in L^{2}\left(\mathbb{R} ; \mathbb{R}^{2}\right)$. Using the embedding $H^{2}(\mathbb{R}) \hookrightarrow L^{\infty}(\mathbb{R})$ and the smoothness of $F(z)$ standard estimates show that the perturbation

$$
H^{2}\left(\mathbb{R} ; \mathbb{R}^{2}\right) \ni z \mapsto F\left(z_{0}+z\right) \in L^{2}\left(\mathbb{R} ; \mathbb{R}^{2}\right)
$$

induces a smooth mapping. In particular the functional derivative

$$
z \mapsto \mathbf{D}\left(F\left(z_{0}+\cdot\right)\right)(z)\langle\zeta\rangle
$$

is continuous from $H^{2}(\mathbb{R})$ to $\mathcal{L}\left(H^{2}(\mathbb{R}) ; L^{2}(\mathbb{R})\right)$.

Finally we consider the terms arising from magnetostatic interaction: Note that

$$
\lambda^{2}(z) B(\kappa, z)=\frac{\partial m_{1}}{\partial z} \sigma(D / \kappa) m_{1}+\frac{\partial m_{2}}{\partial z}(\sigma(D / \kappa)-1) m_{3},
$$

where the components $m_{i}$ have to be considered as functions of $z$. Let $\mathbf{m}^{0}=$ $\mathbf{m}\left[z_{0}\right]$ be the magnetization field associated with a Walker solution with corresponding coordinate vector field $z_{0}$. Let $z$ be any $H^{2}$-perturbation. Let us denote the corresponding variation of the magnetization field by $\mathbf{m}\left[z_{0}+z\right]$. Since the parameterization $\mathbf{m}: \mathbb{R}^{2} \rightarrow \mathbb{R}^{3}$ is smooth, $\left(m_{1}\left[z_{0}\right], m_{3}\left[z_{0}\right]\right) \in L^{2}\left(\mathbb{R} ; \mathbb{R}^{2}\right)$, and $H^{2}\left(\mathbb{R}^{2}\right) \hookrightarrow L^{\infty}(\mathbb{R})$, we have a smooth correspondence

$$
H^{2}\left(\mathbb{R} ; \mathbb{R}^{2}\right) \ni z \mapsto\left(m_{1}\left[z_{0}+z\right], m_{3}\left[z_{0}+z\right]\right) \in L^{2}\left(\mathbb{R} ; \mathbb{R}^{2}\right) .
$$

Likewise, the mappings

$$
\begin{gathered}
H^{2}\left(\mathbb{R} ; \mathbb{R}^{2}\right) \ni z \mapsto\left(\frac{\partial m_{1}}{\partial z}\left[z_{0}+z\right], \frac{\partial m_{3}}{\partial z}\left[z_{0}+z\right]\right) \in L^{\infty}\left(\mathbb{R} ; \mathbb{R}^{2} \times \mathbb{R}^{2}\right) \\
\text { and } H^{2}\left(\mathbb{R} ; \mathbb{R}^{2}\right) \ni z \mapsto \lambda^{-2}\left(z_{0}+z\right) \in L^{\infty}(\mathbb{R})
\end{gathered}
$$

are smooth and induce smooth correspondences with multiplication operators on $L^{2}(\mathbb{R})$. Thus, in view of (34) the continuity of $(\kappa, z) \mapsto B(\kappa, z)$ is a consequence from the following fact.

Lemma 1. The mapping $\Phi: \mathbb{R} \times L^{2}(\mathbb{R}) \rightarrow L^{2}(\mathbb{R})$ defined by

$$
\Phi(\kappa, w)=\sigma(D / \kappa) w \text { for } \kappa \neq 0 \quad \text { and } \Phi(0, w)=w
$$

is continuous, and we have $\|\Phi(\kappa, w)\|_{L^{2}} \leq\|w\|_{L^{2}}$ for every $\kappa \in \mathbb{R}$. 
Proof. Suppose $\kappa_{\varepsilon} \rightarrow \kappa_{0}$ in $\mathbb{R}$ and $w_{\varepsilon} \rightarrow w_{0} \in L^{2}(\mathbb{R})$. Then $0 \leq \sigma\left(\xi / \kappa_{\varepsilon}\right) \leq 1$ and $\sigma\left(\xi / \kappa_{\varepsilon}\right) \rightarrow \sigma\left(\xi / \kappa_{0}\right)$ for every $\xi \neq 0$ with the convention that $\sigma\left(\xi / \kappa_{0}\right)=1$ if $\kappa_{0}=0$. Thus we have in frequency space

$$
\begin{gathered}
\int\left|\sigma\left(\xi / \kappa_{\varepsilon}\right) \hat{w}_{\varepsilon}(\xi)-\sigma\left(\xi / \kappa_{0}\right) \hat{w}_{0}(\xi)\right|^{2} d \xi \leq 2 \int\left|\hat{w}_{\varepsilon}(\xi)-w_{0}(\xi)\right|^{2} d \xi \\
+2 \int\left|\hat{w}_{0}(\xi)\right|^{2}\left|\sigma\left(\xi / \kappa_{0}\right)-\sigma\left(\xi / \kappa_{\varepsilon}\right)\right|^{2} d \xi
\end{gathered}
$$

where the first integral tends to zero by $L^{2}$-convergence of $w_{\varepsilon}$ while the second by dominated convergence using the bounds and pointwise convergence of the multiplier $\sigma\left(\xi / \kappa_{\varepsilon}\right)$ for every $\xi \neq 0$.

Let us show that the functional derivative with respect to the vector field $z$

$$
(z, \kappa) \mapsto \mathbf{D}\left(B\left(\kappa, z_{0}+\cdot\right)\right)(z) \quad, \quad H^{2}(\mathbb{R}) \times \mathbb{R} \rightarrow \mathcal{L}\left(H^{2}(\mathbb{R}) ; L^{2}(\mathbb{R})\right)
$$

is continuous. In view of (34), the chain rule for functional derivatives, and what we have shown before, this is a consequence of the following general observation: Let $\Psi: \mathbb{R}^{2} \rightarrow \mathbb{R}$ be smooth and $z_{0}: \mathbb{R} \rightarrow \mathbb{R}^{2}$ be bounded. Then

$$
\mathbf{D}\left(\sigma(\xi / \kappa) \Psi\left(z_{0}+\cdot\right)\right)(z)\langle\zeta\rangle=\sigma(\xi / \kappa)\left(\nabla \Psi\left(z_{0}+z\right) \cdot \zeta\right) \text {. }
$$

Now since $z \mapsto \nabla \Psi\left(z_{0}+z\right)$ is continuous as a mapping from $H^{2}(\mathbb{R})$ to the space of linear maps acting on $L^{2}\left(\mathbb{R}^{2} ; \mathbb{R}^{2}\right)$ via multiplication, Lemma 1 yields the continuity of $(\kappa, z) \mapsto \mathbf{D}\left(\sigma(\xi / \kappa) \Psi\left(z_{0}+\cdot\right)\right)(z)$ as an operator on $L^{2}(\mathbb{R})$.

On the other hand $\kappa \mapsto \mathcal{G}((z, c), \kappa)$ is not uniformly differentiable. Indeed, the derivative of the mapping $\kappa \mapsto \sigma(D / \kappa) u$ becomes singular as $\kappa \rightarrow 0$ in the sense that it approaches $-\frac{1}{2}(-\Delta)^{-1 / 2} u$, which is unbounded in $L^{2}(\mathbb{R})$. We observe a singular perturbation phenomenon, and this in turn will restrict the regularity of $z$ as an implicit function of $\kappa$, when using the implicit function theorem.

\section{Linearization and non-degeneracy}

Here we study the linearization $\mathcal{L}_{0}=\mathbf{D}_{1} \mathcal{G}(0,0)$, where $\mathbf{D}_{1}$ denotes the partial functional derivative with respect to $(z, c)$, about the pulled-back Walker solution 
$z_{0}(x)=\left(0, v_{0}(x)\right)$ with associated propagation speed $c_{0}$. Recall that both $z_{0}$ and $c_{0}$ as well as the coordinate system depend on $H$ which is supposed to be fixed, but needs to be restricted in size when proving non-degeneracy. For our convenience we denote the conformal factor along the Walker solution $z_{0}=\left(0, v_{0}\right)$ by $\lambda\left(v_{0}(x)\right)=2 /\left(1+v_{0}(x)\right)^{2}$.

\section{The exchange (Dirichlet) term}

First we consider the non-linear operator $N$ coming from Dirichlet energy (exchange energy), given as the covariant $x$-derivative of $\partial_{x} z$ along $z$, i.e.

$$
N(z)=-D_{x} \partial_{x} z=-\partial_{x}^{2} z-\Gamma(z)\left\langle\partial_{x} z, \partial_{x} z\right\rangle,
$$

where

$$
\Gamma(z)\left\langle\partial_{x} z, \partial_{x} z\right\rangle=\lambda(z)\left|\partial_{x} z\right|^{2} z-2 \lambda(z)\left(z \cdot \partial_{x} z\right) \partial_{x} z .
$$

We shall determine the linearization $\mathbf{D} N\left(z_{0}\right)$ at the Walker solution $z_{0}$ for fixed field strength. Standard calculations, repeatedly using that $z_{0}=\left(0, v_{0}\right)$, show that the cross-terms vanish. We have

$$
\begin{array}{r}
\mathbf{D} N_{1}\left(z_{0}\right) u=\left[-\partial_{x}^{2} u+b_{1}(x) \partial_{x} u+r_{1}(x) u, 0\right], \\
\quad \mathbf{D} N_{2}\left(z_{0}\right) v=\left[0,-\partial_{x}^{2} v+b_{2}(x) \partial_{x} v+r_{2}(x) v\right]
\end{array}
$$

where the coefficients $r_{i}(x)$ and $b_{i}(x)$, being multiples of $\partial_{x} v_{0}$ and $\left|\partial_{x} v_{0}\right|^{2}$, respectively, decay rapidly as $|x| \rightarrow \infty$. Recall that in our coordinate representation all Walker solutions $z_{0}$ for admissible $H$ only differ by a scaling factor. Thus all corresponding coefficients $b_{i}(x)$ and $r_{i}(x)$ agree up to a prefactor and a scaling factor.

For later purpose, a more detailed knowledge about the coefficient $r_{1}(x)$ is needed. We have

$$
r_{1}(x)=-\lambda\left(v_{0}(x)\right)\left|\partial_{x} v_{0}(x)\right|^{2}
$$

which can be expressed as a function of $v_{0}(x)$. Indeed, from (19) we deduce that

$$
\left|\mathbf{m}_{0}^{\prime}(x)\right|^{2}=\lambda^{2}\left(v_{0}(x)\right)\left|\partial_{x} v_{0}(x)\right|^{2}=\left(1+\sin ^{2} \varphi\right)\left(1-\lambda^{2}\left(v_{0}(x)\right) v_{0}^{2}\right),
$$

so that

$$
-\lambda\left(v_{0}(x)\right) r_{1}(x)=\left(1+\sin ^{2} \varphi\right)\left(1-\lambda^{2}\left(v_{0}(x)\right) v_{0}^{2}\right)
$$


and we define a function $d=d(v)$ such that $r_{1}(x)=d\left(v_{0}(x)\right)$. A short calculation using that $\sin \varphi=\mathcal{O}(H)$ (see property (b) of Walker solutions in Section 4) shows that

$$
d(v)=-\frac{1}{4} \lambda(v)\left(1-v^{2}\right)^{2}+\mathcal{O}(H) .
$$

\section{The linearized operator}

Recall that for fixed $z: \mathbb{R} \mapsto \mathbb{R}^{2}$ and $c \in \mathbb{R}$ the function $\mathcal{G}((z, c), \kappa)$ has three components $\mathcal{G}_{i}, i=1,2,3$. Thus $\mathbf{D}_{1} \mathcal{G}((z, c), \kappa)=\partial_{(z, c)} \mathcal{G}((z, c), \kappa)$ is a $3 \times 3$ operator-valued matrix. The components of $\mathcal{G}$ arising from applied and magnetostatic field interaction clearly depend on the field strength $H$ partially through the gauging of our coordinate system. Also the pulled-back Walker solution $z_{0}$ and $c_{0}$ depend on $H$ through a scaling factor or the inclination angle, respectively. Thus also the linearization will clearly carry this dependence, but this will be suppressed in our notation.

We first consider the diagonal entries $L_{1}=\left.\frac{\partial \mathcal{G}_{1}}{\partial u}\right|_{(0,0)}$ and $L_{2}=\left.\frac{\partial \mathcal{G}_{2}}{\partial v}\right|_{(0,0)}$ which will be dominant ingredients in the proof non-degeneracy. Note that from the definition of $\mathcal{G}$ the third diagonal entry vanishes.

$$
\begin{gathered}
L_{1} u=-\partial_{x}^{2} u+\left(b_{1}(x)+\alpha c_{0}\right) \partial_{x} u+\left(d\left(v_{0}(x)\right)+f\left(v_{0}(x)\right)\right) u, \\
\text { where } \quad f(v)=\frac{1}{4} \lambda^{2}(v)\left(1+4 v^{2}+3 v^{4}\right)+\mathcal{O}(H) \\
\text { and } d(v)=-\frac{1}{4} \lambda(v)\left(1-v^{2}\right)^{2}+\mathcal{O}(H)
\end{gathered}
$$

The coefficients $b_{1}(x)$ and $d\left(v_{0}(x)\right)$ arise from the exchange (Dirichlet) term (see above), while $f\left(v_{0}(x)\right)$ comes from anisotropy, the induced and the applied field via

$$
f(v)=\frac{\partial}{\partial u}\left(F_{1}(\cdot)+B_{1}(0, \cdot)\right)(0, v) .
$$

The second diagonal entry has the following form

$$
\begin{gathered}
L_{2} v=-\partial_{x}^{2} v+\left(b_{2}(x)+\alpha c_{0}\right) \partial_{x} v+\left(r_{2}(x)+g\left(v_{0}(x)\right)\right) v, \\
\text { where } g(v)=\frac{1}{4} \lambda^{2}(v)\left(v^{4}+4 v^{2}-1\right)+\mathcal{O}(H) .
\end{gathered}
$$


The rapidly decaying coefficients $b_{2}(x)$ and $r_{2}(x)$ arise from the exchange (Dirichlet) term (see above), while $g\left(v_{0}(x)\right)$ comes from anisotropy, the induced and the applied field via

$$
g(v)=\frac{\partial}{\partial v}\left(F_{2}(\cdot)+B_{2}(0, \cdot)\right)(0, v)
$$

We have the following off-diagonal components:

$$
M_{1} u=\frac{\partial \mathcal{G}_{2}}{\partial u}(0,0) u=c_{0} u^{\prime}+h\left(v_{0}(x)\right) u \quad \text { and } \quad M_{2} v=\frac{\partial \mathcal{G}_{1}}{\partial v}(0,0) v
$$

where in view of (32)

$$
h(v)=\frac{\partial}{\partial u}\left(F_{2}(\cdot)+B_{2}(0, \cdot)\right)(0, v)=\mathcal{O}(H) .
$$

For later analysis the first order operator $M_{2}$ is only needed to be uniformly bounded, and we can skip a more detailed description. The derivation of the remaining entries is immediate. Finally we arrive at the following operator matrix

$$
\mathcal{L}_{0}=\left[\begin{array}{ccc}
L_{1} & M_{2} & -v_{0}^{\prime} \\
M_{1} & L_{2} & \alpha v_{0}^{\prime} \\
0 & \delta_{0} & 0
\end{array}\right]
$$

representing $\mathbf{D}_{1} \mathcal{G}(0,0)$. We should show that, at least for small field strength $H$, the operator $\mathcal{L}_{0}: X \rightarrow Y$ is bounded and has a bounded inverse on the perturbation spaces $X$ and $Y$. It turns out that (38) can be considered as a weakly coupled $2 \times 2$ system, that can be solved by the following Schur type argument:

Lemma 2. Given bounded linear operators $T_{1}: X_{1} \rightarrow Y_{1}, S_{1}: X_{1} \rightarrow Y_{2}$, $S_{2}: X_{2} \rightarrow Y_{1}$, and $T_{2}: X_{2} \rightarrow Y_{2}$ on Banach spaces. Suppose that $T_{1}$ and $T_{2}$ have a bounded inverse, respectively, and

$$
\left\|S_{1}\right\|\left\|S_{2}\right\|\left\|T_{1}^{-1}\right\|\left\|T_{2}^{-1}\right\|<1, \quad \text { then } \quad \mathcal{L}=\left[\begin{array}{cc}
T_{1} & S_{2} \\
S_{1} & T_{2}
\end{array}\right]: X_{1} \times X_{2} \rightarrow Y_{1} \times Y_{2}
$$

has a bounded inverse $\mathcal{L}^{-1}$ on the product space.

Proof. Let $(f, g) \in Y_{1} \times Y_{2}$ and consider the associated equations $T_{1} x+S_{2} y=f$ and $T_{2} y+S_{1} x=g$. Since $T_{2}$ is invertible we can write $y=T_{2}^{-1}\left(g-S_{1} x\right)$. Thus the first equation can be written as

$$
\left(\mathbf{1}-T_{1}^{-1} S_{2} T_{2}^{-1} S_{1}\right) x=T_{1}^{-1}\left(f-S_{2} T_{2}^{-1} g\right) \in X_{1} .
$$


The right-hand side defines a bounded linear operator from $Y_{1} \times Y_{2}$ to $X_{1}$, while the operator on the left-hand side is invertible on $X_{1}$ if $\left\|T_{1}^{-1} S_{2} T_{2}^{-1} S_{1}\right\|<1$.

According to (38) we define the operators $T_{i}$ and $S_{i}$ for $i=1,2$ to be

$$
T_{1}=L_{1}, \quad T_{2}=\left[\begin{array}{cc}
L_{2} & \alpha v_{0}^{\prime} \\
\delta_{0} & 0
\end{array}\right], \quad S_{1}=\left(\begin{array}{c}
M_{1} \\
0
\end{array}\right), \quad \text { and } \quad S_{2}=\left(M_{2},-v_{0}^{\prime}\right)
$$

with target spaces $Y_{1}=L^{2}(\mathbb{R})$ and $Y_{2}=L^{2}(\mathbb{R}) \times \mathbb{R}$ and domains $X_{1}=\operatorname{dom} T_{1}=$ $H^{2}(\mathbb{R})$ and $X_{2}=\operatorname{dom} T_{2}=H^{2}(\mathbb{R}) \times \mathbb{R}$. Then $X$ and $Y$ are the corresponding product spaces. In this setting we shall show:

Proposition. There is a number $H_{0}>0$ such that for $|H|<H_{0}$ the operators $T_{1}$ and $T_{2}$ have a uniformly bounded inverse. Moreover, the operators $S_{1}$ and $S_{2}$ are bounded, and $\left\|S_{1}\right\|$ can be made arbitrarily small by choosing $H$ sufficiently small.

Thus for sufficiently small $|H|<H_{0}$ Lemma 2 applies, and we conclude that $\mathcal{L}_{0}=\mathbf{D}_{1} \mathcal{G}(0,0)$ has a bounded inverse in this regime. Recall also that $\mathcal{G} \in$ $C(X \times \mathbb{R} ; Y)$ and $\mathbf{D}_{1} \mathcal{G} \in C(X \times \mathbb{R} ; \mathcal{L}(X ; Y))$. Thus we infer from the continuous version of the implicit function theorem, see e.g. [8] Theorem 3.4.10, for fixed $|H|<H_{0}$ the existence of a threshold $\kappa(H)>0$ and a continuous correspondence $\kappa \mapsto\left(z_{\kappa}, c_{\kappa}\right)$ such that $\mathcal{G}\left(\left(z_{\kappa}, c_{\kappa}\right), \kappa\right)=0$ for all $|\kappa|<\kappa(H)$ proving the theorem we announced in the introduction.

\section{Proof of the Proposition}

Many of the arguments that follow are well-known from the spectral theory of ordinary differential equations and have been used before in the context of nondegeneracy and stability, cf. [1], [5], [7].

The operator $T_{1}$. We show that $L_{1}$ is uniformly invertible for small $H$. For this purpose we consider the coefficient $d\left(v_{0}(x)\right)+f\left(v_{0}(x)\right)$ for $v$ in (35). A short calculation shows that

$$
d(v)+f(v)=\frac{1}{4} \lambda(v)\left(1+8 v^{2}-v^{4}\right)+\mathcal{O}(H) \geq \frac{1}{2}+\mathcal{O}(H)
$$

which is positive provided $H$ is sufficiently small. Then it is easy to see that 0 cannot be an eigenvalue for $L_{1}$, and clearly the same holds for the (formal) adjoint 
since the essential spectrum does not reach the origin (see e.g. [5] page 140). Thus, for fixed but small $H$ and for each $f$, the equation $L_{1} u=f$ has a unique solution. But obviously, altering $H$ corresponds to a continuous perturbation in the operator norm. To get an uniform bound on the inverse in $|H|<H_{0}$ for some threshold field $H_{0}>0$ we need the following abstract fact (see [7] for related stability results):

Lemma 3. Let $T: X \rightarrow Y$ be bounded and invertible. Then there are open neighborhoods $\mathcal{U}$ of $T$ and $\mathcal{V}$ of $T^{-1}$ in the space of bounded linear operators such that the inversion map

$$
\mathcal{I}: \mathcal{U} \ni A \mapsto A^{-1} \in \mathcal{V}
$$

exists as a smooth diffeomorphism in the topology of bounded operators.

Proof. It is enough to find a smooth correspondence between operators $A$ close to $T$ and their right-inverse. Indeed, since the set of bounded invertible operators is open, and, as shown by simple algebraic manipulations, a right-inverse necessarily agrees with the uniquely determined inverse, we might simply choose the sets $\mathcal{U}$ and $\mathcal{V}$ smaller.

Define the smooth map $\Phi(A, B)=A \circ B-1$. Clearly $\Phi\left(T, T^{-1}\right)=0$ and $\partial_{2} \Phi\left(T, T^{-1}\right)=B \mapsto T \circ B$ has a bounded inverse given by $A \mapsto T^{-1} \circ A$. Thus, by means of the implicit function theorem there is mapping $i$ defined on an open neighborhood of $T$ such that $\Phi(A, i(A))=0$, i.e. assigning a right inverse.

The operator $T_{2}$. We first show that $L_{2}$ and its adjoint $L_{2}^{*}$ have one-dimensional kernels. We differentiate the second component equation

$$
0=\frac{d}{d x} \mathcal{G}_{2}(0,0)(x)=\frac{\partial G_{2}}{\partial v}\left(0, v_{0}\right)\left\langle v_{0}^{\prime}\right\rangle(x)=\left(L_{2} v_{0}^{\prime}\right)(x),
$$

where we have used the fact that $u_{0}=0$. This shows that $v_{0}^{\prime}$ is in the kernel of $L_{2}$. Recall that

$$
L_{2} v=-\partial_{x}^{2} v+\left(b_{2}(x)+\alpha c_{0}\right) \partial_{x} v+\left(r_{2}(x)+g\left(v_{0}(x)\right)\right) v .
$$

We consider the coefficient $r_{2}(x)+g\left(v_{0}(x)\right)$ for $v$. Using the positivity of the null function $v_{0}^{\prime}>0$, the formula for $g(v)$ in (36), and the fact that $r_{2}(x)$ vanishes at infinity, it is easy to see that

$$
\left\{x \in \mathbb{R} \mid r_{2}(x)+g\left(v_{0}(x)\right) \leq 0\right\}
$$


is compact. Thus, by means of a contradiction argument based on the uniqueness for the Cauchy problem, one can prove that any null function other than $v_{0}^{\prime}$ must be linearly dependent.

This implies in turn that $L_{2}^{*}$ also has a one-dimensional kernel. Indeed, we might choose a suitable parametrix $P: L^{2}(\mathbb{R}) \rightarrow H^{2}(\mathbb{R})$ for $L_{2}$, e.g. $P=\left(-\partial_{x}^{2}+\right.$ $\left.\beta(x) \partial_{x}+1\right)^{-1}$ where $\beta(x)=b_{2}(x)+\alpha c_{0}$ will suffice. Then it is not difficult to see that $P L_{2}=\mathbf{1}+K$, where $K: L^{2}(\mathbb{R}) \rightarrow L^{2}(\mathbb{R})$ is compact by means of the compact embedding $H^{2}(\mathbb{R}) \hookrightarrow L^{2}(\mathbb{R})$ (note that $\left(1-\partial_{x}^{2}\right)^{-1}$ has a HilbertSchmidt kernel). Thus $P L_{2}$ is a Fredholm operator of index zero on $L^{2}(\mathbb{R})$ which implies that $\operatorname{dim} \operatorname{ker} P L_{2}=\operatorname{dim} \operatorname{ran}\left(P L_{2}\right)^{\perp}=\operatorname{dim} \operatorname{ker} L_{2}^{*} P^{*}$, and consequently $\operatorname{dim} \operatorname{ker} L_{2}=\operatorname{dim} \operatorname{ker} L_{2}^{*}$. A simple calculation shows that a corresponding null function for $L_{2}^{*}$ is given by

$$
\psi(x)=\exp \left(-\int_{0}^{x} \beta(y) d y\right) v_{0}^{\prime}(x)
$$

where $\beta(x)=b_{2}(x)+\alpha c_{0}$. We still need to show that $\psi$ is admissible. We claim that $\psi$ is rapidly decaying when $H$ is small enough. Since $b_{2}(x)$ is rapidly decaying we have $\psi(x) \leq C \exp \left(-\alpha c_{0} x\right) v_{0}^{\prime}$ for some constant $C>0$. But from (26) we deduce that

$$
v_{0}^{\prime}(x)=\frac{1}{2}\left(1+\sin ^{2} \varphi\right)^{1 / 2} \operatorname{sech}^{2}\left[\frac{1}{2}\left(1+\sin ^{2} \varphi\right)^{1 / 2} x\right] \leq c \exp (-\sqrt{2}|x|) .
$$

This implies the claim since $c_{0}=\mathcal{O}(H)$ by property (c) of Walker solutions (see Section 4). In particular the integral $\int v_{0}^{\prime} \psi_{0} d x$ exists and is positive.

Now we are in the position to show invertability of $T_{2}$. Note that the equation $T_{2}(u, c)=(f, b)$ that we intend to solve uniquely for arbitrary $f \in L^{2}(\mathbb{R})$ and $b \in \mathbb{R}$ reads as follows:

$$
\begin{aligned}
L_{2} v+c \alpha v_{0}^{\prime} & =f \\
v(0) & =b
\end{aligned}
$$

Now the first equation is, by means of the Fredholm alternative, solvable provided

$$
\int f \psi_{0} d x=-c \alpha \int v_{0}^{\prime} \psi_{0} d x
$$

which fixes $c$ and determines $v$ up to a multiple of $v_{0}^{\prime}$. But since $v_{0}^{\prime}$ is positive, the second equation yields uniqueness. We conclude that for fixed but small $H$ the 
operator $T_{2}$ is invertible. Uniform invertability for small $H$ again follows from Lemma 3.

The operators $S_{1}$ and $S_{2}$ are of lower order with uniformly bounded coefficients and obviously bounded. For $u \in H^{1}(\mathbb{R})$ and $c \in \mathbb{R}$ we have in view of (37)

$$
S_{1}(u, c)=\left(M_{1} u, 0\right)=c_{0} u^{\prime}+h\left(v_{0}\right) u \quad \text { where } h(v)=\mathcal{O}(H) .
$$

Hence

$$
\left\|S_{1}(u, c)\right\|_{Y_{2}} \leq c_{0}\left\|u^{\prime}\right\|_{L^{2}}+\left\|h\left(v_{0}\right)\right\|_{L^{\infty}}\|u\|_{L^{2}} \leq \mathcal{O}(H)\|(u, c)\|_{X_{1}}
$$

since for the propagation speed of the Walker solution $c_{0}=\mathcal{O}(H)$. See property (c) in Section 4.

Acknowledgments.

I would like to thank Professor Hans Weinberger for supporting this research and for many important comments and suggestions on the subject matter.

\section{References}

[1] P. W. Bates, P. C. Fife, X. Ren, and X. Wang, Traveling Waves in a Convolution Model for Phase Transitions, Arch. Rational Mech. Anal. 138 (1997).

[2] A. DeSimone, R. V. Kohn, S. Müller, and F. Otto, Magnetic microstructure a paradigm of multiscale problems, Proc. ICIAM 99 (Edinburgh), Eds. J.M. Ball and J.C.R. Hunt, (1999).

[3] P. C. Fife, Mathematical Aspects of Reacting and Diffusing Systems, Lecture Notes in Biomathematics 28, Springer-Verlag, Berlin-Heidelberg-New York (1979).

[4] C. J. Gracía Cervera, Magnetic Domains and Magnetic Domain Walls, Ph.D. thesis, New York University (1999).

[5] D. Henry, Geometric Theory of Parabolic Equations, Lecture Notes in Mathematics 840, Springer-Verlag, Berlin-Heidelberg-New York (1981). 
[6] A. Hubert and R. Schäfer, Magnetic Domains, The Analysis of Magnetic Microstructures, Springer-Verlag, Berlin-Heidelberg-New York (1998).

[7] T. Kato, Perturbation Theory for Linear Operators, Grundlehren der mathematischen Wissenschaften 123, Springer-Verlag, Berlin-Heidelberg-New York (1966).

[8] S. G. Krantz and H. R. Parks, The Implicit Function Theorem, History, Theory, and Applications, Birkhäuser, Boston (2002).

[9] L. D. Landau and E. Lifshitz, On the theory of the dispersion of magnetic permeability in ferromagnetic bodies, Phys. Z. Sovietunion 8 (1935).

[10] C. Melcher, The logarithmic tail of Néel walls in thin films, Arch. Rational Mech. Anal. 168 (2003).

[11] L.R. Walker, unpublished. Reported in J. F. Dillon Jr., Domains and Domain Walls, in Magnetism, Eds. G. R. Rado and H. Suhl, Academic Press, New York (1963). 